\title{
X-ray analysis of polycrystalline aluminium subjected to fatigue cycling
}

\author{
KALYANI VIJAYAN, A MANI, C BALASINGH and A K SINGH \\ Materials Science Division, National Aeronautical Laboratory, Bangalore 560017 , India \\ MS received 4 March 1988

\begin{abstract}
Variations in the halfwidth values of X-ray reflections from fatigue-cycled, polycrystalline aluminium samples have been analysed. An oscillatory variation of the halfwidths with fatigue cycling has been observed. Analysis of the diffraction line profiles indicates that broadening arises mainly because of the build-up of microstrains during fatigue cycling. The present data indicate that (i) broadening due to fatigue cycling increases with glancing angle; (ii) changes in halfwidth and integral widths, due to fatigue cycling, are comparable and (iii) $\left(b / b_{0}\right)$ versus $N$ curves for fatigue cycling under constant stress amplitude and flight loading conditions are comparable.
\end{abstract}

Keywords. Aluminium; fatigue cycling; X-ray diffraction.

\section{Introduction}

It is well known that the shape and position of an X-ray diffraction profile depend on microstructural parameters such as crystallite size, residual stress, microstrain, faultings etc. Changes in the microstructural parameters due to fatigue cycling of the specimen, manifest as changes in the characteristics of X-ray diffraction profiles. Based on this correlation, many investigations on the fatigue behaviour of polycrystalline materials have been carried out (Taira and Honda 1960, 1961a, 1962; Baggerly and Pelloux 1967; Taira et al 1968, 1969; Ohuchida and Iwasaki 1971; Kurobe et al 1973; Pangborn et al 1981).

The parameters commonly used to characterize diffraction profiles are FWHM (full width at half maximum), also referred to as halfwidth $b$, integral width $\beta$, variance and Fourier coefficients (Klug and Alexander 1954). Although the three parameters integral width, variance and Fourier coefficients are considered more reliable than halfwidth, the latter is more easily derived, especially when computercontrolled diffraction equipments are not available. A large number of fatigue investigations based on the halfwidth values are reported in literature (Taira and Honda 1960, 1961a, 1961b; Taira et al 1963, 1968; Ohuchida and Iwasaki 1971; Ohumori et al 1974; Pangborn et al 1981; Takechi et al 1981; Kuo and Cohen 1983). The results indicate that the variation of the ratio $\left(b / b_{0}\right)$ with $N$ could be represented by a generalized three-stage curve. Here $b_{0}$ and $b$ are the respective halfwidths of a Bragg reflection, before and after $N$ fatigue cycles. A typical three-stage curve for an annealed specimen, for which the halfwidths are measured from the near-surface region, is schematically shown in figure 1 (curve a). Pangborn et al (1981) reported that the $\left(b / b_{0}\right)$ vs $N$ plot results in a straight line, if the diffraction line is recorded from the interior of the specimen by using short wavelength radiation.

In this paper, we report the results on the fatigue behaviour of polycrystalline aluminium samples, obtained primarily from the halfwidth values. The most important aspect of these results concerns an oscillatory variation observed in the halfwidth of diffraction lines with progressive increase in the number of fatigue cycles. A preliminary report of this hitherto unknown oscillatory variation was 


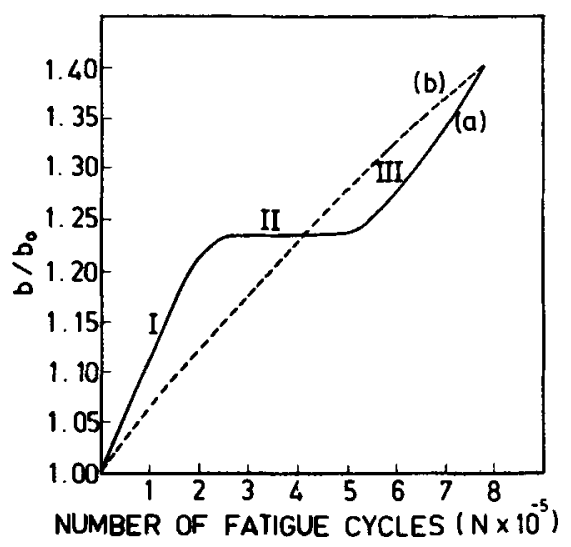

Figure 1. (a) A typical, three-stage $\left(b / b_{0}\right)$ vs $N$ curve when the diffraction patterns are recorded from the near-surface region of the specimen. The three stages are marked as I, II and III respectively. (b) Variation of $\left(b / b_{0}\right)$ with $N$ when penetrating radiation is used (Pangborn et al 1981).

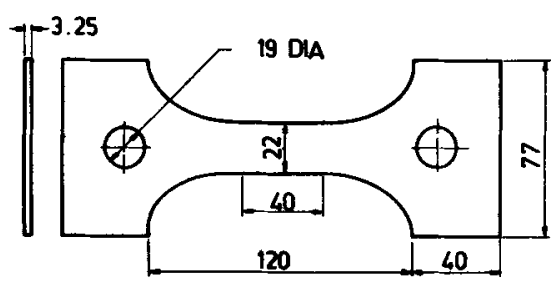

Figure 2. Geometry and dimensions (in $\mathrm{mm}$ ) of the specimen.

published (Vijayan et al 1986). In addition to the oscillatory variation, results describing (i) the angular dependence of profile broadening, (ii) the sensitivities of integral and halfwidths to fatigue cycling, (iii) the fatigue behaviour under flight loading conditions and (iv) the correlation of the halfwidths with microstrains derived from line profile analysis are now reported. Scanning electron micrographs have also been used to analyse the oscillatory variation in halfwidth values.

\section{Experimental details}

Specimens with the geometry shown in figure 2 were machined from commercial purity (99.2\%) ALFAX aluminium sheets. The $19.1 \mathrm{~mm}$ dia holes along the length of the specimen were incorporated such that the specimens could be assembled in a special jig (figure 3) which permits simultaneous loading of two specimens on the fatigue testing machine. To prevent initiation of cracks near the assembling holes, the regions surrounding them were strengthened by cold working which was achieved by forcing a tapered pin of appropriate dimensions through the holes, using a hydraulic press.

The machined specimens were annealed at $160^{\circ} \mathrm{C}$ for $1 \mathrm{~h}$. The gauge section was initially hand polished using 600 grit emery. The central portion of the gauge section which was exposed to X-rays was then electropolished for $2 \mathrm{~min}$ using $75 \%$ 

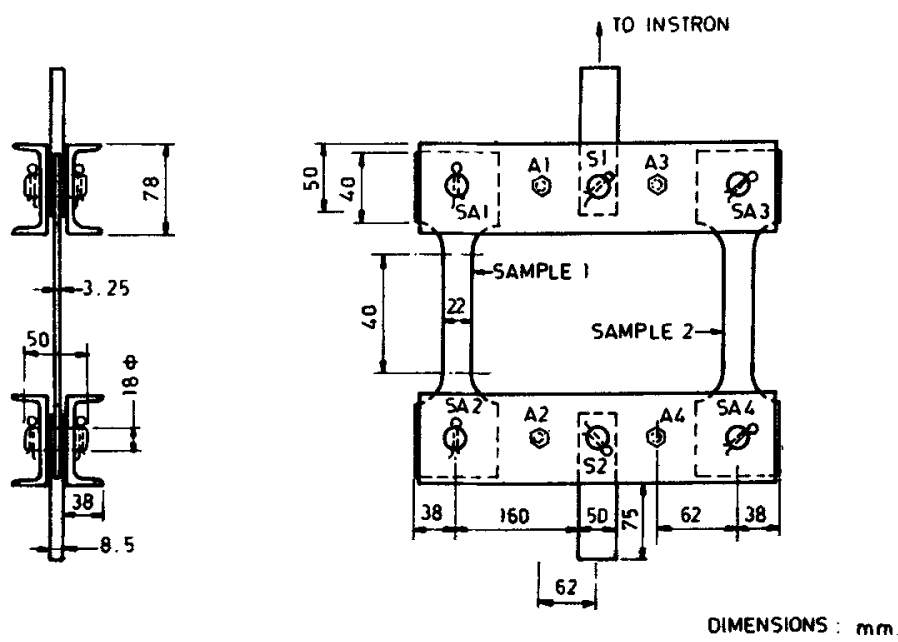

Figure 3. Schematic representation of the jig used for simultaneous loading of two specimens.

methanol and $25 \%$ nitric acid maintained at $10^{\circ} \mathrm{C}$. Before commencing the fatigue cycling, back reflection powder photographs were recorded on a flat film. The uniformity of the intensity distribution along the diffraction ring indicated the absence of texture in the specimens.

The fatigue cycling was conducted on an Instron-1343 unit equipped with automatic servohydraulic load maintainer. The aluminium specimens were axially loaded in the tension-tension mode. Two different types of load procedures were adopted: (i) fatigue cycling at constant stress amplitudes and (ii) fatigue cycling under spectrum or flight loading conditions. Fatigue cycling under constant stress amplitude corresponds to repeated, periodic application of a specific stress amplitude. Spectrum loading, on the other hand, corresponds to the application of a large number of varying stress amplitudes in a single loading of the specimen. The sequence and magnitudes of the stress amplitudes in such a spectrum have been chosen (Sunder et al 1984) to resemble those experienced by a combat aircraft and hence the name, flight loading. In figure 4 , the load patterns corresponding to both constant stress amplitude loading and flight loading are schematically shown.

Fatigue cycling experiments were carried out at a frequency of $15 \mathrm{~Hz}$ and at constant stress amplitudes of 32,39 and $46 \mathrm{MPa}$ respectively. The ratio of minimum to maximum stress was always maintained at $0 \cdot 1$. The fatigue cycling was interrupted at chosen intervals, and X-ray diffraction patterns were recorded on a Philips powder diffractometer using $\mathrm{CuK} \alpha$ radiation, proportional counter and a graphite monochromator in the diffracted beam. The diffraction profiles were recorded using a sample rotation speed of $1 / 8^{\circ}$ per min and a chart speed of $20 \mathrm{~mm}$ per min. The halfwidths were measured on the recorded profiles. The integral widths were obtained by measuring the areas under the profiles, using a AD-380 graphics system. In these experiments, the choice of the radiation restricted the examination to only the near-surface region of the specimens.

The fatigue cycling and X-ray experiments were conducted over a period of about 8 months. It was therefore essential to periodically check the alignment of the 

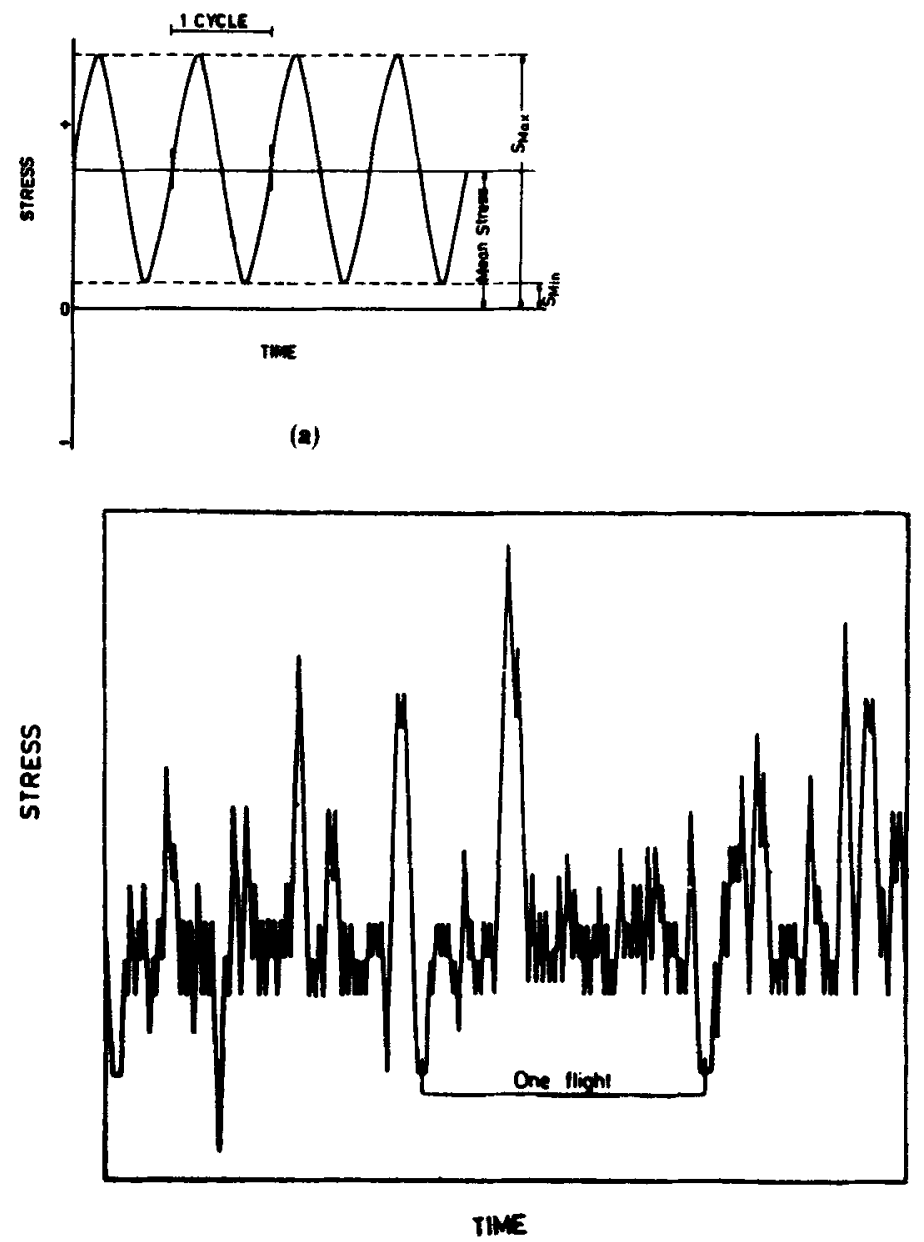

(b)

Figure 4. (a) Schematic representation of the load pattern for constant stress amplitudes. $S_{\max }$ and $S_{\min }$ represent the maximum and minimum values of the stress amplitude in each load cycle. (b) Schematic representation of the load pattern for flight loading.

diffractometer. This was done by recording the (422) reflection from an aluminium specimen prepared and polished as mentioned earlier; this specimen was never fatigue-cycled. The halfwidths of the profiles recorded periodically (once a week), from this specimen remained constant over the entire duration of the experiments. The estimated standard deviation, $\sigma$, of the halfwidth measurement was $1.8 \%$.

In a few cases, the halfwidths were measured at different places along the gaugelength of $40 \mathrm{~mm}$, on both sides of the specimen. It was found that within experimental errors, the halfwidths remained constant along the gauge length and also on either side of the specimen indicating that the specimens were being loaded symmetrically. The data presented in this paper were, however, obtained by recording the diffraction line from only one surface of the specimen.

The crystallite size $(D)$ and the microstrains $(E)$ at various stages of fatigue cycling were calculated using the single line method (Keijser et al 1982). In a limited number 
of cases, the multiple line analysis was also performed (Klug and Alexander 1954). In the single line method, it is assumed that the Cauchy and the Gaussian components represent respectively the contribution from crystallite size and the microstrains. The $f$ profile which only includes the effect of fatigue cycling was calculated from the profile obtained on fatigue-cycled specimens by removing the contribution due to instrumental factors. In this analysis, the profile of the (422) reflection was used.

In the multiple line method (Klug and Alexander 1954), a Cauchy-Gaussian approximation has been used to describe the diffraction profile. The values $D, E$ and $\beta$ are related by the expression

$$
\beta^{2} / \tan ^{2} \theta=(K \lambda / D)(\beta / \tan \theta \sin \theta)+16 E^{2},
$$

where $\theta$ is the Bragg angle and $\lambda$ the wavelength of $\mathrm{X}$-rays used. $K$, the Scherrer constant, is numerically close to unity. The values of $D$ and $E$ can be obtained respectively from the slope and the intercept of a line fitted through the $(\beta / \tan \theta)^{2}$ vs $(\beta / \tan \theta \sin \theta)$ data points.

The multiple line method is often used to calculate the crystallite size and strain with reference to a specific crystallographic direction. In such cases, only a reflection and its higher orders are considered while fitting equation (1). In the present analysis, we have used the reflections (200), (220), (311), (222), (400) and (422), because of the difficulty in obtaining a reflection and atleast two higher orders with $\mathrm{CuK} \alpha$ radiation. The $D$ and $E$ values obtained by this procedure represent the average over various crystallographic directions.

\section{Results and discussion}

\subsection{Dependence of broadening on the Bragg angle}

The nature of the angular dependence of halfwidth is well established (Cagliotti et al 1958). However, data on the angular dependence of the additional broadening introduced by fatigue cycling are very limited and are also conflicting. Results obtained by Pangborn et al (1981) on Al2024 alloy indicate that the width due to fatigue cycling increases with glancing angle. On the other hand, results of Taira et al (1963) on $0.07 \%$ carbon steel show that during fatigue cycling the width of the low angle reflection (200) is higher than the width of the higher angle reflections (211), (220) and (310). Further, the overlap and the cross-over observed in Taira et al's $\left(b / b_{0}\right)$ vs $N$ curves for various reflections indicate that the angular dependence of the broadening varied with the progress of fatigue cycling. The reported variation does not appear to have any systematic character.

In the case of the ALFAX specimens, the angular dependence of the broadening introduced by fatigue cycling was established by measuring the integral widths of the observable reflections. With the monochromator in position on the Philips powder diffractometer, reflections beyond $2 \theta=140^{\circ}$ could not be recorded. Therefore, only the reflections with $2 \theta \leqslant 140^{\circ}$ were used. Also, as the intensities of the reflections (400) and (222) were low, the width measurements were not sufficiently reliable and these two reflections were not included in this investigation. The $\left(\beta / \beta_{0}\right)$ vs $N$ curves for various reflections are presented in figure 5 . Here $\beta_{0}$ refers to the integral width of a reflection, before fatigue cycling. It is found that for the ALFAX specimens, the width 


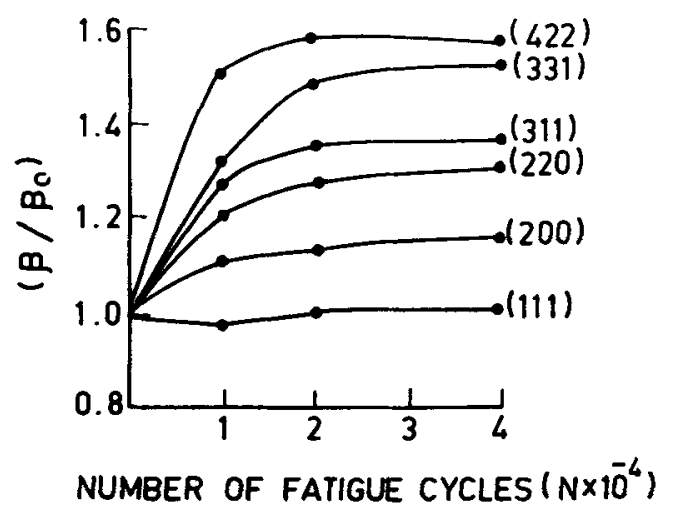

Figure 5. $\left(\beta / \beta_{0}\right)$ vs $N$ curves for various reflections from ALFAX specimens showing angular dependence.

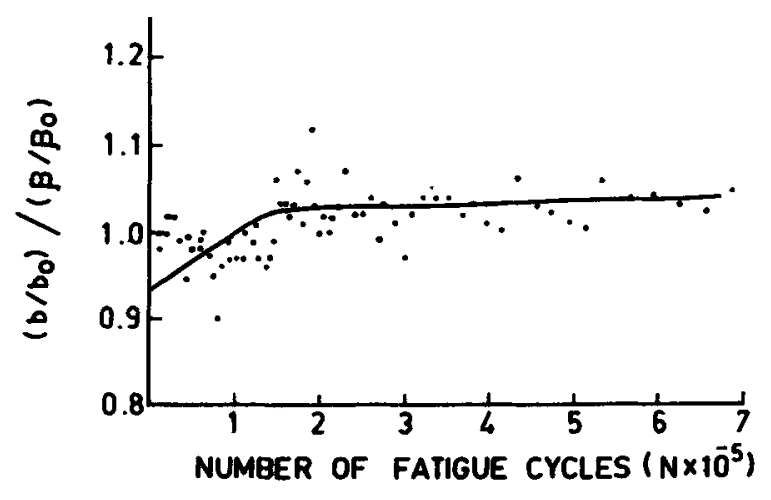

Figure 6. Variation of $\left(b / b_{0}\right) /\left(\beta / \beta_{0}\right)$ with $N$.

due to fatigue cycling increased with glancing angle. Further, the absence of any overlap or cross-over among the $\left(\beta / \beta_{0}\right)$ vs $N$ curves suggests that unlike the $0.07 \%$ carbon steel, the angular dependence of the profile broadening of ALFAX specimens did not vary with the progress of fatigue cycling. Based on these results, (422) was identified as the reflection exhibiting the highest width due to fatigue cycling. Hence, in the ensuing analysis, the widths of only this reflection were measured. The results presented in the subsequent sections pertain only to the (422) reflection.

\subsection{Integral vs halfwidth values}

In order to compare sensitivities of $\beta$ and $b$, values of the integral and halfwidths of the (422) reflection were estimated at various stages of fatigue cycling under constant stress amplitude. Figure 6 shows the variation of the ratio $\left(b / b_{0}\right) /\left(\beta / \beta_{0}\right)$ with $N$. The marked slope of the curve in the early stages of fatigue cycling indicates a higher sensitivity of the halfwidth values. However, with further fatigue cycling, the curve is nearly parallel to the $N$-axis, indicating that with the progress of fatigue cycling, the sensitivities of $b$ and $\beta$ tend to become comparable. 


\subsection{Fatigue cycling under flight loading conditions}

The data collected from ALFAX specimens show that as in the case of fatigue cycling under constant stress amplitude, the fatigue behaviour under flight loading conditions is also described by a three-stage $\left(b / b_{0}\right)$ vs $N$ curve. In figure 7 , a typical curve obtained under flight loading conditions is presented. Here, $N$ refers to the number of flights. The curve corresponding to fatigue cycling under constant stress amplitude of $42.5 \mathrm{MPa}$ is also included in figure 7 as an inset. Comparison of the curves shows that the $\left(b / b_{0}\right)$ vs $N$ curve under flight loading is similar to that under constant stress amplitude loading. Further, the $\left(b / b_{0}\right)$ value at fatigue failure is independent of the loading procedure and is perhaps characteristic of the specimen.

It may be mentioned here that Taira and Honda (1961a) have studied the behaviour of steel, fatigue-cycled at various constant stress amplitudes. Their results indicate that for cycling under constant stress amplitude, the value of $\left(b / b_{0}\right)$ at fatigue failure is independent of the applied stress amplitude.

\subsection{Variation of $\left(b / b_{0}\right)$ with $N$}

It is observed that most of the $\left(b / b_{0}\right)$ vs $N$ curves reported in literature and those in figure 5 have been obtained by measuring the halfwidth values at widely spaced intervals of $N$. The chosen intervals are atleast $15 \%$ of the fatigue lives of the respective samples.

Data from ALFAX specimens have been collected by interrupting the fatigue cycling at finer intervals of $N$, which are of the order of 2 to $5 \%$ of the fatigue life $N_{f}$. In figure $8(a)$, the $\left(b / b_{0}\right)$ vs $N$ curve obtained at such fine intervals, for the applied stress amplitude of $32 \mathrm{MPa}$, is presented. In contrast to the commonly reported three-stage curve (figure 1$)$, where, in each stage, the $\left(b / b_{0}\right)$ values are shown to vary in a smooth and linear fashion, the curve in figure 8 (a) exhibits an oscillatory variation. It may be mentioned that the curve in figure $8(\mathrm{a})$ is obrained by joining adjacent experimental points by straight lines. If values of two or more adjacent points differed by less than the estimated standard deviation, $\sigma$, of the $b$

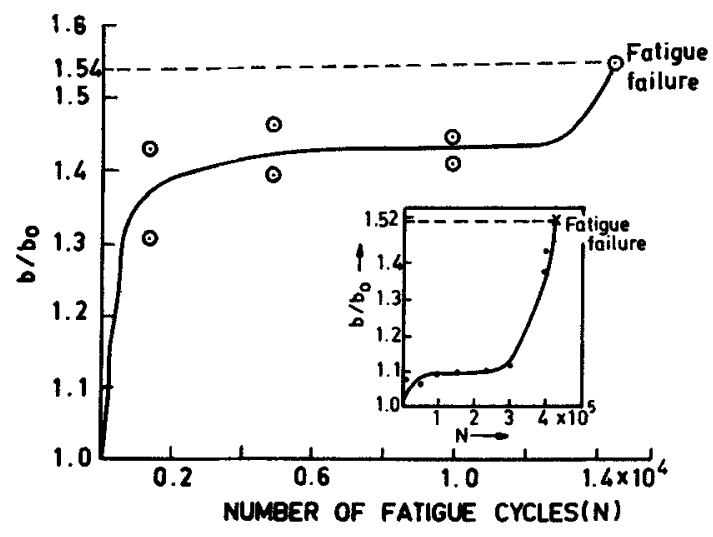

Figure 7. $\left(b / b_{0}\right)$ vs $N$ curve obtained under flight loading conditions. Curve in the inset represents the $\left(b / b_{0}\right)$ vs $N$ curve corresponding to loading at constant stress amplitude of 42.5 MPa. 


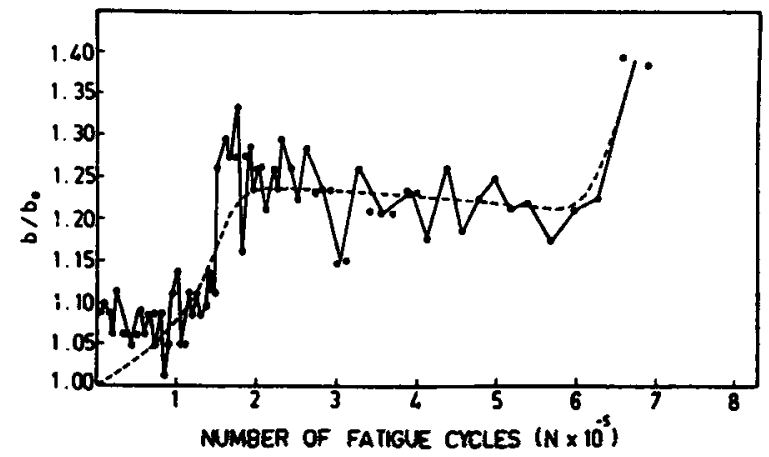

(a)

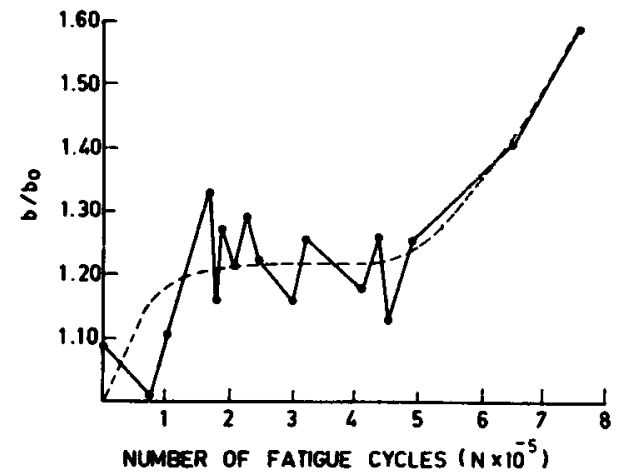

(b)

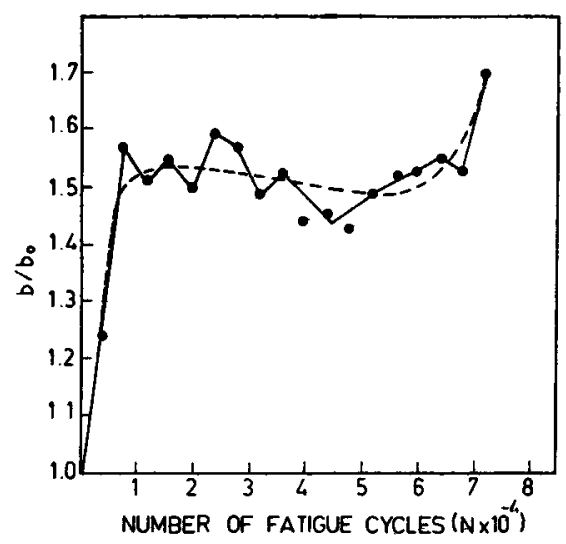

(c)

Figure 8. (a) $\left(b / b_{0}\right)$ vs $N$ data at $32 \mathrm{MPa}$. Adjacent points differ by atleast $\sigma$. Dashed line represents the average curve. (b) $\left(b / b_{0}\right)$ vs $N$ data at $32 \mathrm{MPa}$. Adjacent points differ by atleast $3 \sigma$. Dashed line represents the average curve. (c) $\left(b / b_{0}\right)$ vs $N$ data at $46 \mathrm{MPa}$. Dashed line represents the average curve.

measurement, then these points are replaced by a single point at the mean position. Thus, adjacent points joined by straight line in figure 8 (a) differ by atleast $\sigma$. The oscillatory nature of the $\left(b / b_{0}\right)$ vs $N$ curve was found to persist even when adjacent points differing by atleast $3 \sigma$.were used. Figure $8(\mathrm{~b})$ represents the curve obtained at the $3 \sigma$ level.

It may be noted that if a few points in the early stages of fatigue cycling are neglected (figure 8) and a mean curve (shown by dashed lines in figure 8) is drawn through the remaining points, the mean curve resembles closely the conventional three-stage curve. This feature suggests that the oscillations could be described as the fine structure of a curve, which, on an average, resembles the three-stage curve. In order to detect the fine structure, it is essential to determine the halfwidth values at sufficiently closely-spaced intervals of $N$. Detection of the oscillations in the present experiments is attributed, primarily, to the fine interval chosen for measuring the halfwidth. The smooth variation characterizing the three-stage $\left(b / b_{0}\right)$ vs $N$ curves reported so far in literature is obviously due to the lack of resolution resulting from the choice of widely-spaced intervals of $N$, used for measuring the halfwidth values.

Occurrence of oscillations is independent of the applied stress amplitude. The 
presence of oscillations in the $\left(b / b_{0}\right)$ vs $N$ curves corresponding to stress amplitudes of 32 and $46 \mathrm{MPa}$ is shown in figures 8 (a) and (c) respectively. In all the cases, the oscillations are statistically significant and the mean curve in each case closely resembles the three-stage curve.

The oscillations start at very early stages of fatigue cycling and persist till fatigue failure occurs. Absence of oscillations in stage I of the curve in figure $8(\mathrm{c})$ corresponding to the higher stress amplitude of $46 \mathrm{MPa}$ is due to the inappropriate choice of the interval used in this stage. It may be pointed out that when the applied stress amplitude is increased, the length of each stage (along the $N$ axis), is shortened (Taira and Honda 1961c). For the stress amplitude of $46 \mathrm{MPa}$, the interval at which the halfwidth was measured in stage I was comparable to the entire span of that stage, and thus lacks the resolution required for detecting the oscillations.

The oscillations are aperiodic. The lack of periodicity has been ascertained by using a fast Fourier transform routine. In this procedure, the program deconvolutes the experimental $\left(b / b_{0}\right)$ vs $N$ curve and estimates the amplitude and period of the component curves. It was found that the $\left(b / b_{0}\right)$ vs $N$ curves shown in figure 8 do not consist of any periodic component.

The amplitudes of the oscillations exhibit a wide range of variation. The highest observed amplitude is as large as $10 \sigma$.

\subsection{Estimation of microstrains}

The single line procedure was carried out using the half and the integral widths of the (422) profiles recorded at various stages of fatigue cycling at constant stress amplitude of $32 \mathrm{MPa}$. Results of this analysis indicate that throughout the fatigue cycling range, the Gaussian component $\beta_{G}^{f}$ of the integral width was conspicuously larger in magnitude than the corresponding Cauchy component $\beta_{c}^{f}$ of the $f$-profile. The average value 17.6 of the ratio $\left(\beta_{G}^{f} / \beta_{c}^{f}\right)$ provides strong evidence that the width of the $f$-profile is essentially from the Gaussian component and that the contribution from the crystallite dimension is small. Hence, in further discussions presented in this section, the crystallite size effect on the width is not included. It was also found that some of the $\beta_{C}^{f}$ values, though small were negative in sign. Negative width of the profile is, however, physically meaningless. The negative value of $\beta_{C}^{f}$ can occur when the $D$ values are so large that the $\beta_{c}^{f}$ 's are of the order of the error in the estimation of the integral widths.

Figure 9 shows the variation of the microstrain values with $N$. The $E$ vs $N$ curve is strikingly similar to the corresponding $\left(b / b_{0}\right)$ vs $N$ curve in figure $8(a)$. The $E$ 's exhibit an oscillatory distribution about a mean line. The mean line is characterized by the presence of three stages marked in figure 9. The average $E$ values of $0.15 \times 10^{-3}, 0.30 \times 10^{-3}$ and $0.40 \times 10^{-3}$ corresponding to stages I, II and III of the $E$ vs $N$ curve point out that the microstrain values increase with the progress of fatigue cycling.

Values of $D$ and $E$ obtained by the multiple line method are listed in table 1. These values correspond to stage $I$ of fatigue cycling at a constant stress amplitude of $39 \mathrm{MPa}$. The $E$ values averaged over various crystallographic directions are of the same order as those obtained for the [422] direction. The average dimension of the crystallite is found to be approximately $1100 \AA$.

The strain distribution shown in figure 9 may be satisfactorily explained by 


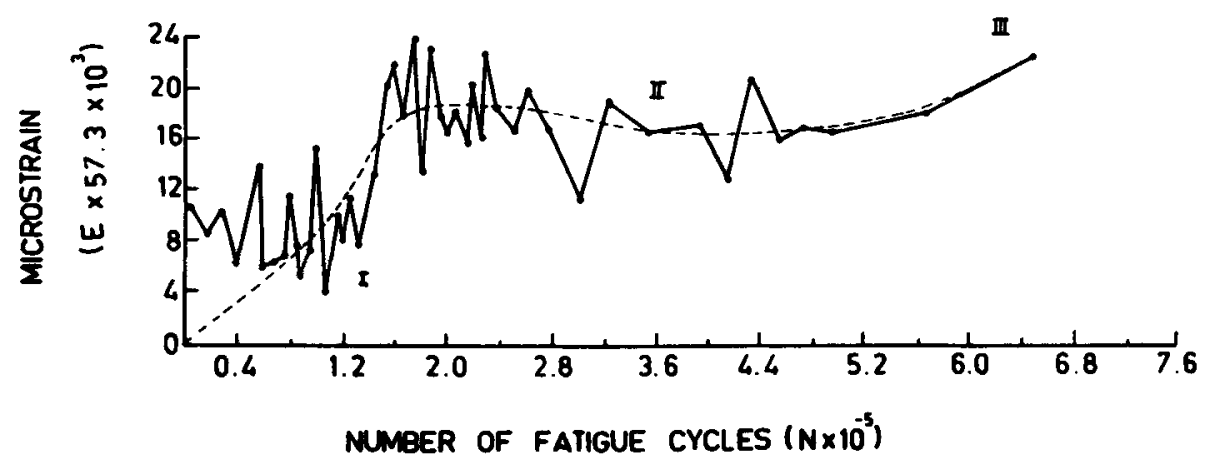

Figure 9. Variation of microstrain $E$ with $N$.

Table 1. Values of $E$ and $D(\AA)$ at various stages of fatigue cycling at constant stress amplitude of $39 \mathrm{MPa}$.

\begin{tabular}{lcc}
\hline $\begin{array}{l}\text { No. of cycles } \\
N \times 10^{-5}\end{array}$ & $\begin{array}{c}\text { Microstrain } \\
E \times 10^{3}\end{array}$ & $\begin{array}{c}\text { Average } \\
\text { crystallite } \\
\text { dimension } \\
D(\AA)\end{array}$ \\
\hline 0.04 & 0.18 & 1341 \\
0.12 & 0.25 & 1025 \\
0.16 & 0.17 & 1255 \\
0.20 & 0.21 & 1081 \\
0.30 & 0.25 & 1032 \\
\hline
\end{tabular}

introducing the concept of microcracks. It is suggested that during fatigue cycling, microcracks are formed when the microstrain values are large enough, as for a peak in the $E$ vs $N$ curve. However, with the formation of microcracks, stress relaxation occurs and consequently the strain values decrease and reach the adjacent troughs in the $E$ vs $N$ curve. The $E$ distribution in figure 9 may therefore be considered to represent the formation and progress of microcracks. The microcracks appear to be formed from early stages of fatigue cycling. The aperiodicity and the varying amplitudes of oscillations may also be taken to represent the random formation of microcracks of varying lengths.

Direct evidence for the formation of microcracks has been obtained from scanning electron micrographs. In figures $10(a)$ and (b) the micrographs recorded from samples fatigue cycled to stages I and III are reproduced. Presence of microcracks in both (a) and (b) confirms that microcracks are formed in stage I itself and persist till stage III. It should, however, be pointed out that for samples in stage I, only a few microcracks are observed on the polished surface. Samples in stage III are found to contain large number of microcracks of the type shown in figure 10(b). Also, for samples in stage III, the microcracks are of larger dimensions.

Our results show that the $\left(b / b_{0}\right)$ vs $N$ curves with $\mathrm{CuK} \alpha$ radiation are oscillatory. These, however, correspond to the near-surface region. The presence or otherwise of oscillations in the curves corresponding to a penetrating radiation like $\mathrm{MoK} \alpha$, is yet to be examined. 


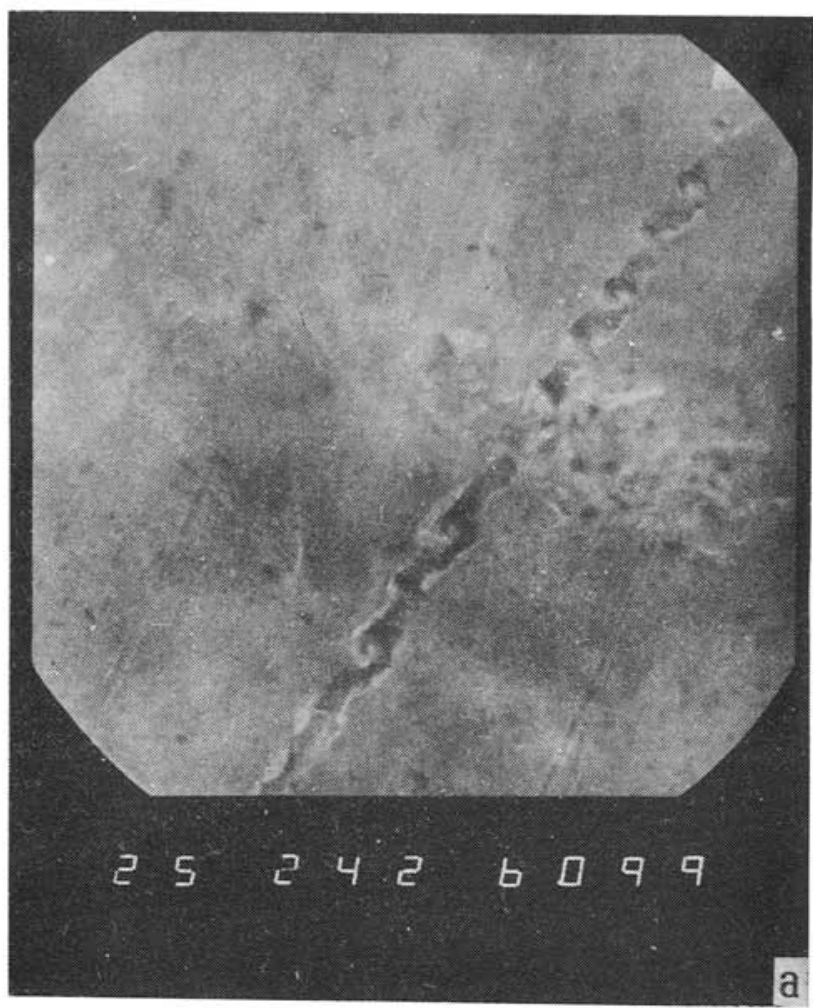

Figure 10. Scanning electron micrograph of sample fatigue cycled to (a) stage I and

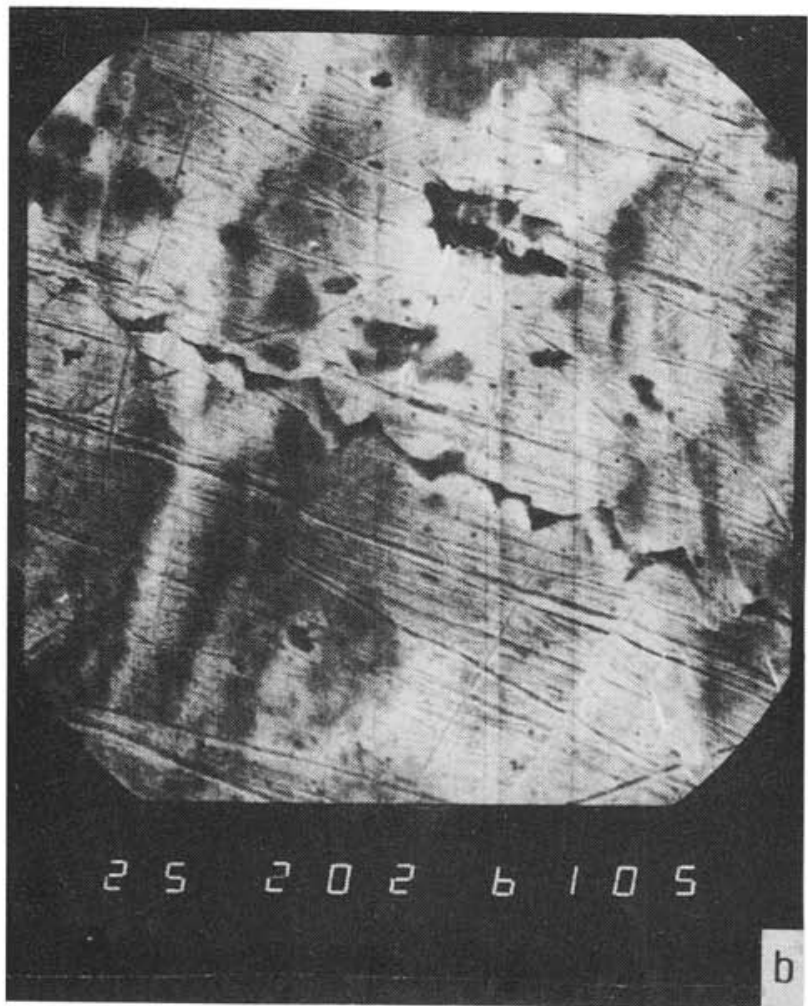
(b) stage III 


\section{Acknowledgement}

The authors thank Dr R Sunder and his group for assistance rendered in using the Instron and $\mathrm{Mr}$ M A Venkataswamy for recording the scanning electron micrographs. They also acknowledge Mr Ramakrishna's help in using the FFT routine.

\section{References}

Baggerly R G and Pelloux R M N 1967 Adv. X-ray Anal. 10328

Cagliotti G, Paoletti A and Ricci F P 1958 Nucl. Instrum. 3223

Keijser Th H, Longford J I, Mittemeijer E J and Vogels A B P 1982 J. Appl. Cryst. 15308

Klug H P and Alexander L E $1954 X$-ray diffraction procedures (New York: John Wiley)

Kuo H K and Cohen J B 1983 Mater. Sci. Eng. 61127

Kurobe T, Ueda K and Wakashima H 1973 Proc. Jpn. Congr. Test Mater. 16164

Ohuchida H and Iwasaki T 1971 Proc. Jpn. Congr. Test Mater. 1429

Ohumori M, Himoto A and Chiba T 1974 Proc. Jpn. Congr. Test Mater. 1761

Pangborn R N, Weissmann S and Kramer I R 1981 Metall. Trans. A12 109

Pangborn R N, Weissmann S and Kramer I R 1981 Adv. X-ray Anal. 24203

Sunder R, Seetharam S A and Bhaskaran T A 1984 Int. J. Fat. 6147

Taira S and Honda K 1960 Proc. Jpn. Congr. Test Mater. 356

Taira S and Honda K 1961a Proc. Jpn. Congr. Test Mater. 417

Taira S and Honda K 1961b Bull. JSME. 4230

Taira S and Honda K 1961c Proc. Jpn. Congr. Test Mater. 4230

Taira S and Honda K 1962 Proc. Jpn. Congr. Test Mater. 58

Taira S, Haysashi K and Tanaka K 1968 Proc. Jpn. Congr. Test Mater. 1125

Taira S, Goto T and Nakano Y 1969 Proc. Jpn. Congr. Test Mater. 128

Taira S, Honda K and Abe T 1963 Proc. Jpn. Congr. Test Mater. 610

Takechi H, Namba K, Fujiwara K and Kawasaki K 1981 Trans. ISII 2192

Vijayan K, Mani A, Balasingh C and Singh A K 1986 Scr. Metall. 201767 